\author{
D. Jaquet - S. Deghmoun - D. Chevenne - D. Collin • \\ P. Czernichow $\cdot$ C. Lévy-Marchal
}

\title{
Dynamic change in adiposity from fetal to postnatal life is involved in the metabolic syndrome associated with reduced fetal growth
}

Received: 22 November 2004 / Accepted: 22 December 2004 / Published online: 15 April 2005

(C) Springer-Verlag 2005

\begin{abstract}
Aims/hypothesis: The aims of this study were to establish the role of insulin resistance in the metabolic syndrome associated with restricted fetal growth and to characterise the fetal and postnatal determinants responsible for the long-term metabolic outcome. Methods: The study population consisted of adults selected on birth data from a maternity registry and born either small for gestational age (SGA) $(n=734$, birthweight $<$ tenth percentile) or appropriate for gestational age (AGA) $(n=886,25$ th $<$ birthweight $<75$ th percentile) and in whom clinical and metabolic parameters of the metabolic syndrome were measured at 22 years of age. Results: Mean values of all components of the metabolic syndrome significantly differed between the two groups, with the metabolic syndrome observed in $2.3 \%$ of the SGA group and in 4\%o of the AGA group $(p=0.0004)$. In SGA subjects, the upper tertile of fasting insulinaemia was associated with the highest values of systolic $(p=0.001)$ and diastolic $(p=0.02)$ blood pressure, triglyceridaemia $(p=0.005)$ and glycaemia at fasting $(p=0.0001)$ and during OGTT $(p=0.0001)$. In GA subjects, insulin resistance was not related to birthweight itself ( $p=$ $0.26)$, but correlated negatively with BMI at birth $(p=0.03)$ and positively with the subsequent postnatal catch-up in BMI $(p=0.009)$. Conclusions/interpretation: Insulin resistance is the keystone of metabolic syndrome associated
\end{abstract}

\footnotetext{
D. Jaquet $\cdot$ S. Deghmoun - P. Czernichow $\cdot$

C. Lévy-Marchal $(\square)$

INSERM Unit 690, Hôpital Robert Debré, 48 Boulevard Sérurier,

75019 Paris, France

e-mail: clairelm@rdebre.inserm.fr

Tel.: +33-1-40031987

Fax: +33-1-40409195

D. Chevenne

Department of Hormonology and Biochemistry, Hôpital Robert Debré,

Paris, France

D. Collin

Obstetric Department, Hospital of Haguenau,

Haguenau, France
}

with SGA, and its origin should be sought in the fetal development process of adiposity that is responsible for postnatal growth and the later development of insulin resistance.

Keywords Adiposity · Catch-up growth · Insulin resistance $\cdot$ Metabolic syndrome $\cdot$ Small for gestational age $\cdot$ Thinness at birth

Abbreviations AGA: appropriate for gestational age GLM: general linear model - MS: metabolic syndrome QUICKI: quantitative insulin sensitivity check index · SGA: small for gestational age

\section{Introduction}

The association between a low birthweight and the metabolic syndrome (MS) [1-3] or with one of its components has been substantially documented in various populations [4-10]. MS was originally described in humans to cluster with insulin resistance $[11,12]$. It has been postulated that insulin resistance also plays a central and primary role in the metabolic complications associated with reduced fetal growth, but this has never been clearly demonstrated and the natural history of MS in subjects born with a low birthweight remains poorly documented. Therefore, the question arises as to when and how MS emerges in this particular clinical situation.

Our previous data have shown that insulin resistance is detectable at as early as 20 years of age [8]. However, only one-third of young adults born small for gestational age (SGA) seem to be affected. In these individuals insulin resistance cannot be explained by a family history of metabolic diseases or obesity [8]. Therefore the reason for this variable predisposition for insulin resistance should be sought either in specific aspects of SGA, or among postnatal events directly linked to SGA. Postnatal catch-up growth aiming at compensating reduced fetal growth is one of the most evident consequences of SGA observed in nearly $90 \%$ of these children [13]. It has been postulated that the catchup growth process might strongly influence the long-term 
metabolic consequences by promoting obesity in adulthood [14].

The aims of the present study were therefore: (1) to investigate whether MS is detectable in SGA young adults and to assess the role of insulin resistance in this association; and (2) to characterise the fetal and postnatal determinants responsible for the variable predisposition for insulin resistance in subjects born SGA. Data come from a large cohort of young adults born either appropriate for gestational age (AGA) or SGA, which includes more than 700 subjects born SGA, this being defined as a birthweight below the tenth percentile of the distribution according to sex and gestational age.

\section{Subjects and methods}

Subjects Subjects were identified from a population-based registry of the metropolitan area of the city of Haguenau in France. This registry included information about all pregnancies and deliveries occurring in this maternity unit from 1971 to $1985(n=27,366)$ and the degree of ascertainment of this registry was more than $80 \%$ [15]. In this region (northeast France), non-Caucasian subjects represent less than $0.1 \%$.

Local growth standard curves have been estimated from all birth data registered during 1971-1985. Gestational age was determined from the last menstrual period and confirmed by physical examination and ultrasonography when available. The SGA group included all singleton subjects born between 32 and 42 weeks of gestation with birthweight $<$ tenth percentile for sex and gestational age according to the local growth standard curves. Selection of the AGA group was made of singleton subjects born between 32 and 42 weeks of gestation, with birth size between the 25 th and the 75th percentiles and who were the first babies in the registry born immediately after a subject born SGA. Taken together, 1,817 subjects born SGA and 1,817 subjects born AGA between 1971 and 1985 were selected from the registry. Forty-five subjects born SGA were excluded because of aberrant measurements in the registry. Of the remaining 3,589 subjects, 1,620 (45\%) took part in this study between 2000 and 2002. As expected, the number of deaths was significantly higher in the SGA group $(p<0.0001)$. Refusal to participate represented the largest cause of non-participation in the AGA group, whereas the proportion of subjects who could not be contacted was the largest cause in the SGA group. Finally, among all subjects who could have been contacted at the time of the study, the proportion of subjects who agreed to participate was similar in the SGA (65.6\%) and AGA (66.0\%) groups. Among the AGA group, there was no significant difference between subjects who participated and those who did not participate in terms of gestational age, birthweight and birth length. In the SGA group, non-participants had significantly reduced birthweight $(2,606 \pm 355$ vs $2,659 \pm 301 \mathrm{~g}, p=0.0001)$ and length $(46.9 \pm 2.2$ vs $47.6 \pm 2.6 \mathrm{~cm}, p=0.0001)$ than participants when adjusted for sex and gestational age. This difference was mainly due to the extent of SGA in subjects who had died.

Clinical characteristics of the two groups at birth are given in Table 1. Mean gestational ages were similar in both groups and sex distribution did not significantly differ between the SGA and AGA groups. According to the selection criteria, subjects born SGA were significantly lighter, shorter and thinner at birth than subjects born AGA when corrected for gestational age and sex.

Study design Subjects who agreed to participate attended a medical visit at the Hospital of Haguenau. Information about medical history was recorded using a standardised questionnaire. Body weight was measured with a portable scale and height with a wall-mounted stadiometer. Weight for height was assessed as BMI $\left(\mathrm{kg} / \mathrm{m}^{2}\right)$. Waist circumference was measured at the level of the umbilicus and hip circumference at the level of the greater trochanter. Blood pressure was measured in the right arm of seated subjects after 30 min rest using an automated device (Dinamap; Critikon, Neuilly-Plaisance, France) and a cuff of recommended size for the mid-upper arm circumference. Three measurements were made at 1-min intervals and the average of the last two measurements was used in the analysis. Blood samples were collected after an overnight fast for measurement of serum lipids, plasma glucose and serum insulin concentrations. A 75-g OGTT was performed and plasma glucose and serum insulin concentrations were measured 30 and $120 \mathrm{~min}$ after the glucose load.

The study protocol was reviewed and approved by the ethical committee of the University of Paris-St Louis and all subjects gave written informed consent.

Assessment of insulin resistance and definition of metabolic syndrome Insulin resistance was assessed by the fasting serum insulin concentration, the fasting insulin/glucose ratio and the quantitative insulin sensitivity check index (QUICKI) calculated as QUICKI=1/(log[fasting serum insulin concentration]+log[fasting blood glucose]) [16].

According to the ATP III criteria [17], subjects were considered as having MS if they had at least three of any of the following criteria: fasting blood glucose $\geq 6.1 \mathrm{mmol} / 1$, waist circumference $\geq 102 \mathrm{~cm}$ (men) or $88 \mathrm{~cm}$ (women),

Table 1 Clinical characteristics of the small for gestational age (SGA) and appropriate for gestational (AGA) groups at birth

\begin{tabular}{lllc}
\hline & $\begin{array}{l}\text { SGA } \\
(n=734)\end{array}$ & $\begin{array}{l}\text { AGA } \\
(n=886)\end{array}$ & $p$-value \\
\hline Gestational age (weeks) & $40(32-43)$ & $40(32-43)$ & 0.70 \\
Sex (men/women) & $336 / 398$ & $430 / 456$ & 0.30 \\
Birthweight (g) & 2700 & 3400 & $<0.0001$ \\
& $(1130-3080)$ & $(2000-4000)$ & \\
Birth length (cm) & $45(37-53)$ & $50(45-53)$ & $<0.0001$ \\
BMI at birth $\left(\mathrm{kg} / \mathrm{m}^{2}\right)$ & $11.5(7.3-17.8)$ & $13.3(9.9-15.6)$ & $<0.0001$ \\
\hline
\end{tabular}

Results are expressed as median (range). Anthropometric parameters were compared after adjustment for sex and gestational age 
triglycerides $\geq 1.7 \mathrm{mmol} / \mathrm{l}$, HDL-cholesterol $<1.0 \mathrm{mmol} / 1$ (men) or $1.16 \mathrm{mmol} / 1$ (women) and blood pressure $\geq 130 / 85$ $\mathrm{mmHg}$ or treated hypertension.

Laboratory procedures Plasma glucose, total cholesterol, HDL-cholesterol and triglyceride concentrations were measured with enzymatic methods.

Serum insulin concentrations were measured using a double-antibody RIA (ERIA Diagnostics Pasteur, Paris, France). Cross-reactivity with proinsulin and derived metabolites was $<1 \%$. Assay sensitivity was $1.2 \mathrm{pmol} / 1$ and intraand interassays $\mathrm{CV}$ values were 3.8 and $8 \%$ at $48 \mathrm{pmol} / \mathrm{l}$, and 2.4 and $4.8 \%$ at $300 \mathrm{pmol} / 1$.

Statistical analysis All analyses were performed using the SAS statistical package (SAS Institute, Meylan, France). Serum insulin, fasting insulin-to-glucose ratio and serum triglyceride concentrations were log transformed to remove positive skewness.

The association between SGA/AGA status and biological and clinical parameters was tested using Fisher's exact test for qualitative variables, and general linear models (GLMs) for quantitative variables. Analyses were carried out on values adjusted for sex and age for anthropometric parameters, and for all covariates known to significantly influence the components of MS (sex, age, BMI, smoking, oral contraception in women and family history of cardiovascular and metabolic diseases [type 2 diabetes and dyslipidaemia]). WHR was added in a second model and results are given with and without adjustment for WHR. Body height was added as a covariate for the analysis of blood pressure since it is known to positively influence blood pressure in humans $[18,19]$. Statistical interactions between sex and SGA/AGA status were tested in all these models.

The association between the components of the MS and insulin resistance was tested in the SGA and AGA groups divided into tertiles of insulin resistance indices (fasting in- sulin, insulin-to-glucose ratio and QUICKI) using GLM procedures with adjustment for the above-cited covariates. Statistical interactions between insulin-resistance indices and SGA/AGA status were systematically tested.

The effect of clinical characteristics at birth on insulinresistance indices were analysed in the SGA group using a GLM procedure with MS components as dependent variables and sex, current age, current BMI, smoking, oral contraception, family history of metabolic diseases, gestational age, birthweight and ponderal index (or BMI at birth) as explanatory variables.

All anthropometric parameters were expressed as a $z$-score. At birth, $z$-scores were derived from the local growth standards in the registry of Haguenau $(n=27,366)$, corrected for sex and gestational age. At the time of the study; $z$-scores were derived from the AGA group as reference $(n=886)$, corrected for sex. The magnitude of catch-up growth in height and BMI was quantified by the difference between adult values and birth values expressed as a $z$-score. The independent effect of catch-up growth on insulin-resistance parameters was tested after adjustment for sex, current age, current BMI, smoking, oral contraception, family history of metabolic diseases, gestational age and BMI at birth expressed as a $z$-score, using a GLM procedure.

Statistical significance was taken as a $p$-value of 0.05 or less.

\section{Results}

Clinical characteristics Clinical characteristics of the two groups at 22 years of age are summarised in Table 2a. Mean body weight and height remained significantly lower in the SGA group than in the AGA group. Mean BMI was comparable between the two groups, but the mean WHR was significantly higher in the SGA group. Family histories of cardiovascular ( 28 vs $28 \%, p=0.76$ ) and type 2 diabetes
Table 2 Clinical characteristics and components of the metabolic syndrome in adulthood in the small for gestational age (SGA) and appropriate for gestational (AGA) groups

Results are expressed as means $\pm \mathrm{SD}$. $p$-values are given after adjustment for sex and age for anthropometric parameters and after adjustment for sex, age, BMI, smoking, oral contraception in women and family history of cardiovascular and metabolic diseases for the metabolic syndrome parameters. $p$-values after a further adjustment for WHR in addition to the previous covariates are given in parentheses. QUICKI quantitative insulin sensitivity check index

\begin{tabular}{lccc}
\hline & SGA $(n=734)$ & AGA $(n=886)$ & $p$-value \\
\hline a Clinical characteristics & & & \\
Age (years) & $21.9 \pm 3.8$ & $22.1 \pm 3.9$ & 0.50 \\
Bodyweight $(\mathrm{kg})$ & $63.1 \pm 13.0$ & $67.2 \pm 13.6$ & $<0.0001$ \\
Body height $(\mathrm{cm})$ & $167.5 \pm 8.9$ & $172.0 \pm 3.9$ & $<0.0001$ \\
BMI $\left(\mathrm{kg} / \mathrm{m}^{2}\right)$ & $22.4 \pm 4.3$ & $22.6 \pm 3.9$ & 0.37 \\
WHR & $0.81 \pm 0.08$ & $0.80 \pm 0.07$ & 0.02 \\
Systolic blood pressure $(\mathrm{mmHg})$ & $121 \pm 11$ & $120 \pm 11$ & 0.13 \\
Diastolic blood pressure $(\mathrm{mmHg})$ & $63 \pm 10$ & $63 \pm 10$ & 0.37 \\
b Metabolic parameters & & & \\
HDL-cholesterol (mmol/1) & $1.40 \pm 0.36$ & $1.43 \pm 0.35$ & $0.005(0.05)$ \\
Triglycerides (mmol/l) & $1.10 \pm 0.58$ & $1.03 \pm 0.52$ & $0.006(0.002)$ \\
Fasting plasma glucose $(\mathrm{mmol} / \mathrm{l})$ & $4.76 \pm 0.40$ & $4.74 \pm 0.37$ & $0.03(0.03)$ \\
120-min plasma glucose $(\mathrm{mmol} / \mathrm{l})$ & $5.34 \pm 1.21$ & $5.20 \pm 1.13$ & $0.02(0.02)$ \\
Impaired glucose tolerance $(\%)$ & $27 \pm 3.7$ & $14 \pm 1.6$ & 0.01 \\
Fasting serum insulin $(\mathrm{pmol} / \mathrm{l})$ & $33.7 \pm 25.0$ & $30.1 \pm 25.3$ & $0.002(0.001)$ \\
Fasting insulin-to-glucose $\times 10^{-9}$ & $7.0 \pm 5.0$ & $6.4 \pm 5.4$ & $0.001(0.001)$ \\
QUICKI & $0.388 \pm 0.040$ & $0.393 \pm 0.034$ & $0.0003(0.008)$ \\
\hline
\end{tabular}


( 8 vs $7 \%, p=0.51$ ) were similar in both groups, but family history of dyslipidaemia was lower in the SGA group than in the AGA group ( 27 vs $32 \%, p=0.04$ ). Smoking was observed in $36 \%$ of the SGA group and $34 \%$ of the AGA group $(p=0.37)$. the frequency of oral contraception was similar in the SGA and AGA women (58 vs $57 \%, p=0.94$ ).

Systolic and diastolic blood pressure measurements did not significantly differ between the two groups (Table 2a). However, systolic blood pressure became significantly higher in the SGA group when adjusted for body height, known to positively influence blood pressure, in addition to the other confounding factors $[18,19]$ (adjusted means: 122 vs $120 \mathrm{mmHg}, p=0.004$ ).

Metabolic parameters Metabolic characteristics of the two groups are summarised in Table $2 \mathrm{~b}$. Mean values for all metabolic components of MS significantly differed between the SGA and the AGA groups: decreased HDL-cho- lesterol concentration, increased triglyceride concentration, increased plasma glucose concentrations at fasting and 120 min after the glucose load with $3.7 \%$ manifesting impaired glucose tolerance according to the WHO-99 criteria [20] and increased insulin-resistance indices (fasting insulin concentration, fasting insulin-to-glucose ratio and QUICKI). All differences remained statistically significant after a further adjustment for WHR.

According to the ATP III criteria [17], 17 of 734 subjects born SGA were affected by MS vs only three of 886 in the AGA group ( $p=0.0004)$. No interaction between sex and SGA/AGA status was statistically significant, reflecting a similar effect of SGA in men and women.

In both groups, the highest tertile of the QUICKI values, taken as a surrogate marker of insulin resistance, was associated with significantly higher mean value of WHR, systolic and diastolic blood pressures, triglyceride concentrations, plasma glucose concentrations both at fasting and
Fig. 1 Effect of insulin resistance on the metabolic syndrome components in the small for gestational age (SGA) and appropriate for gestational (AGA) groups. Insulin sensitivity was assessed by the quantitative insulin sensitivity check index subsequently divided into tertiles (highest values [1st tertile] are represented as open bars, the second tertile is represented in light grey and the third in dark grey). The highest tertile (lowest values) $(n=3)$ represents the more insulin-resistant subjects. Adjusted means and $p$-values are given after adjustment for current age, sex, BMI, smoking, oral contraception in women, family history of metabolic and cardiovascular diseases. ${ }^{*} p<0.05, * * p<0.01$, $* * * p<0.001$
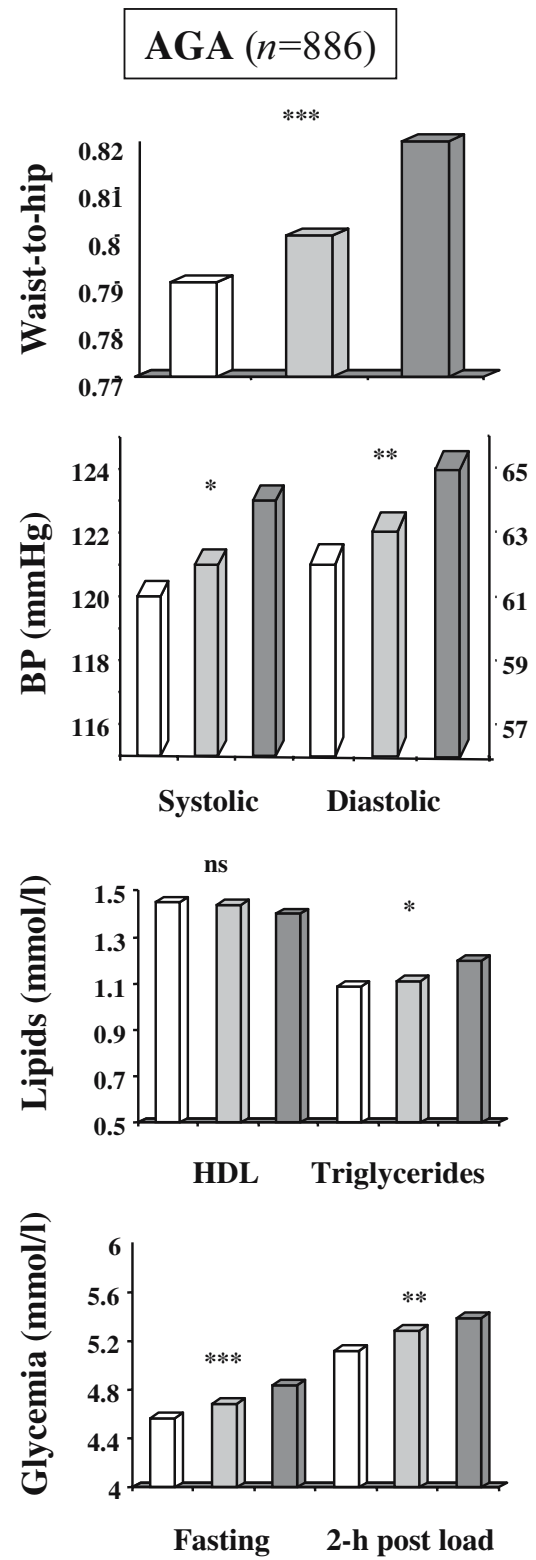
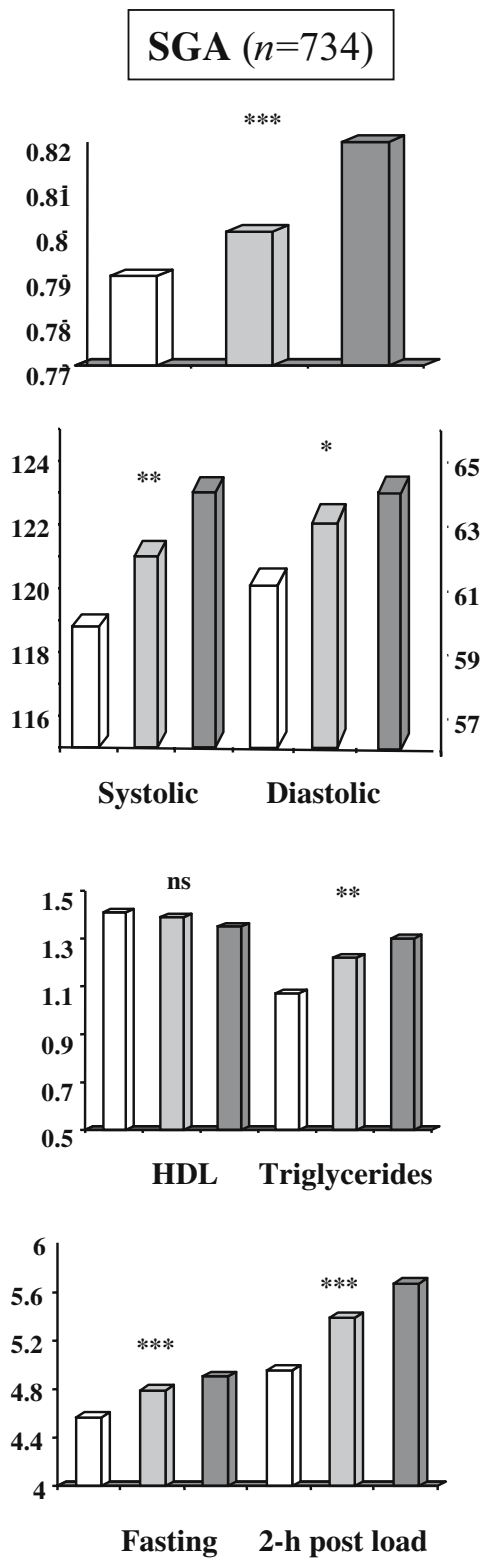
after the glucose load, and lower HDL, although the difference did not reach statistical significance for this latter parameter (Fig. 1). No significant differences (statistical interaction) were observed between subjects born SGA or AGA in the relationship between the QUICKI and WHR $(p=0.64)$, systolic $(p=0.77)$ or diastolic blood pressure $(p=$ $0.31)$, HDL $(p=0.93)$ or triglyceride concentrations $(p=0.14)$ and fasting $(p=0.13)$ or $2 \mathrm{~h}$ postload glycaemia $(p=0.22)$. Similar results were obtained in both groups whatever the surrogate index for insulin resistance (fasting insulin-toglucose ratio and fasting insulinaemia).

Clinical characteristics of SGA at birth and insulin resistance in adulthood Neither birthweight (1,130-3,080 g) nor gestational age (32-42 weeks) influenced fasting insulinaemia ( $p=0.26$ and 0.62 , respectively), the QUICKI ( $p=0.67$ and 0.67 , respectively) or the insulin-to-glucose ratio ( $p=0.28$ and 0.55 , respectively) in adulthood. In contrast, ponderal index $\left(\mathrm{kg} / \mathrm{m}^{3}\right)$ was inversely related to fast-

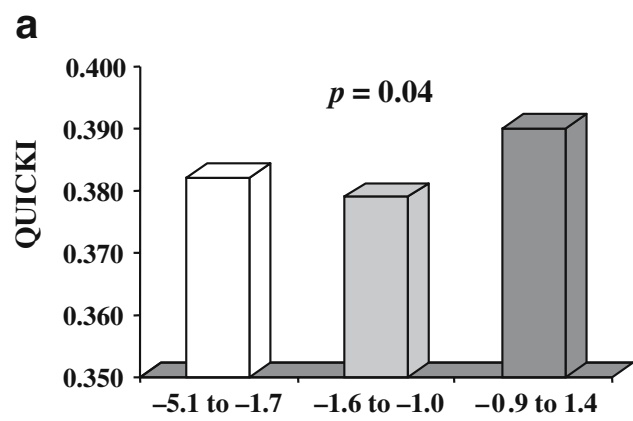

BMI at birth (tertile of $\mathrm{z}$ - score)

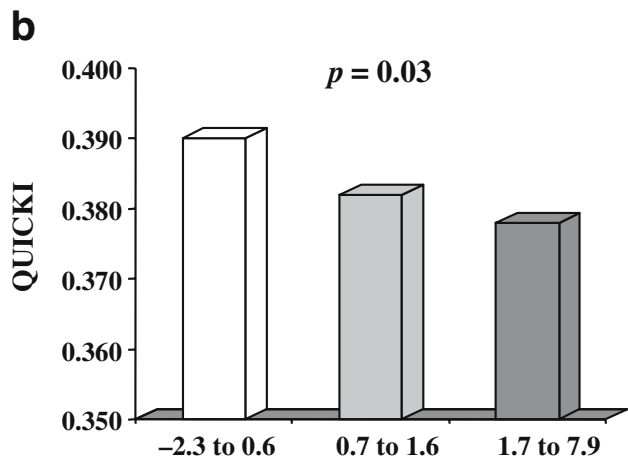

Catch-up in BMI (tertile of $z$ - score)

Fig. 2 Effect of thinness at birth and catch-up of adiposity on insulin resistance in adulthood in subjects born small for gestational age $(n=734)$. a Effect of BMI at birth on the quantitative insulin sensitivity check index (QUICKI) measured in adulthood. BMI at birth was expressed in tertiles of $z$-scores according to sex and gestational age. $p$-values are given after adjustment for sex, current age, adult BMI, smoking, oral contraception in women and family history of cardiovascular and metabolic diseases. b Effect of catch-up in BMI on the QUICKI measured in adulthood. Catch-up in BMI (adult BMI $[z$-score $]-\mathrm{BMI}$ at birth $[z$-score $]$ ) is expressed in tertiles. $p$-values are after adjustment for sex, current age, adult BMI, smoking, oral contraception in women and family history of cardiovascular and metabolic diseases

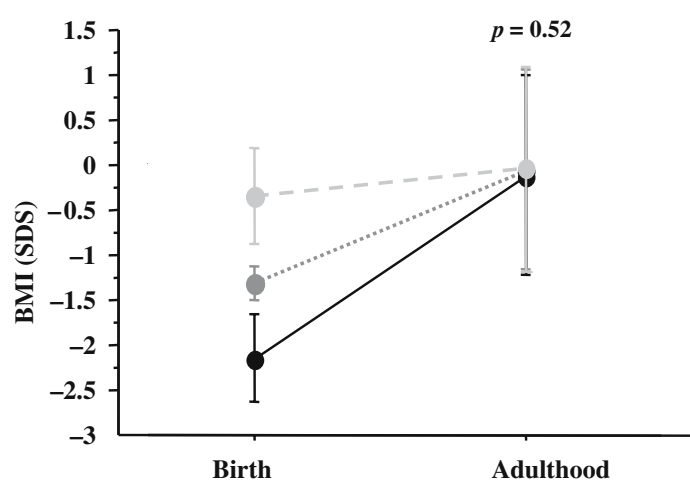

Fig. 3 Catch-up in BMI according to BMI at birth in subjects born small for gestational age $(n=734)$. Subjects were divided into tertiles of BMI at birth expressed in $z$-scores according to sex and gestational age (the lowest tertile is in black, the second tertile in dark grey and the highest in light grey). Adult BMI is expressed in $z$ scores $(S D S)$ according to sex ( $p$-value after adjustment for current age)

ing serum insulin concentration $(p=0.04)$, the insulin-toglucose ratio $(p=0.03)$ and the QUICKI $(p=0.05)$. Similar observations were obtained when thinness at birth was assessed by BMI, which was inversely related to fasting serum insulin concentration $(p=0.03)$, the insulin-to-glucose ratio $(p=0.02)$ and the QUICKI $(p=0.04)$ (Fig. 2a). No such significant effect of ponderal index or BMI at birth was observed on the other parameters of the MS.

Catch-up growth and insulin resistance in subjects born $S G A$ Mean adult height was increased by $+0.75 \pm 1.43 z$ score in comparison with birth length $(p<0.0001)$, but 76 $(10.3 \%)$ subjects showed adult short stature $(<-2 z$-score). Catch-up height was not significantly related to fasting serum insulin concentrations $(p=0.46)$, insulin-to-glucose ratio $(p=0.41)$ or QUICKI $(p=0.60)$ and to the other parameters of MS. The insulin-to-glucose ratios were similar in subjects with either final short stature or adult height $>-2 z$ score $(0.384 \pm 0.04$ vs $0.389 \pm 0.04, p=0.33)$.

In contrast, catch-up in BMI was significantly associated with increased fasting serum insulin concentrations, insulin-to-glucose ratio and QUICKI, even when adult BMI was taken into account ( $p=0.004, p=0.009$ and $p=0.03$, respectively) (Fig. 2b).

As expected, catch-up in BMI was inversely related to BMI at birth $(p=0.0001)$. However, subjects who experienced the larger catch-up did not end up with obesity as young adults (Fig. 3).

\section{Discussion}

In the present study, significant differences were observed as early as 22 years of age in all insulin-resistance indices and in components of MS in subjects born SGA in comparison with subjects born AGA. Additionally, we demonstrate that all components of MS cluster with insulin resistance in subjects born SGA as in subjects born AGA. It should certainly be pointed out that, except for insulin 
resistance itself, differences between the SGA and AGA groups in the components of the syndrome were moderate and probably of minor clinical relevance at this age. This might explain the inconstancy of the association between low birthweight or SGA and the other parameters of MS especially in young subjects $[7,8,10,21,22]$. It is likely that, although the association between insulin resistance and reduced fetal growth is constant, the expression and/or age at onset of the other parameters of the MS depend on the degree of genetic predisposition or on the postnatal environmental circumstances observed in the different study populations. Nonetheless, MS was already observed in $2.3 \%$ of these young adults born SGA, whereas it remained exceptional in their age-matched controls (less than 4\%o). Taken together, our study is the first to confirm the early appearance of MS in young adults born SGA and the primary role of insulin resistance in its appearance.

What determines the development of insulin resistance in the context of reduced fetal growth still remains unclear. In the present study, neither gestational age nor birthweight influences the risk for insulin resistance in young adults born SGA. In contrast, thinness at birth appears to be a major determinant of the later development of insulin resistance. Several epidemiological studies have shown the role of thinness at birth, in addition to low birthweight, on insulin resistance or type 2 diabetes, emphasising therefore the role of impaired fetal growth rather than prematurity to explain the effect of low birthweight $[6,23]$. By studying a large group of subjects with a wide range of adiposity at birth $(-5.1$ to $1.4 z$-score), but all born SGA, we are now able to demonstrate that thinness at birth and not birthweight itself explains the relationship between low birthweight and the long-term metabolic complications. This observation is critical since it gives new insight into the origins of the association between low birthweight and the later development of insulin resistance in adulthood. From a broader point of view, it would be most interesting to see whether the effect of thinness at birth itself is similar in subjects within a normal range of birthweights. Unfortunately, the AGA group of our study is not appropriate for such an analysis because of our stringent selection criteria (birth size [weight and length] between the 25th and the 75 th percentile) leading to small variation of adiposity at birth within a normal range ( -1.3 to $1.4 z$-score).

It has been speculated that catch-up growth, which is observed in the majority of subjects born SGA $[13,24]$ increases the risk for insulin resistance later in life, by promoting obesity in adulthood [14]. A recent study shows that insulin sensitivity was not affected in prepubertal children born SGA with subsequent catch-up growth provided that they showed a current BMI within the normal range [25]. Here, we showed that catch-up height has no influence on insulin resistance in subjects born SGA, which is in agreement with studies reporting that insulin resistance is not related to linear growth in children born SGA $[25,26]$. By contrast, we found a strong effect of catch-up in BMI on insulin resistance in subjects born SGA. However, the positive association between catch-up in BMI and insulin resistance remained statistically independent of adult adiposity. Moreover, catch-up in BMI was significantly related to the degree of thinness at birth and did not seem to favour obesity in adulthood, arguing therefore against the previously proposed hypothesis [14].

These results point therefore to the critical role of thinness at birth on the risk for insulin resistance and MS associated with reduced fetal growth. Previous studies have shown that a low ponderal index in subjects born SGA was associated with a dramatically reduced body fat mass at birth $[27,28]$. Therefore, thinness at birth can mainly be seen as the reflection of the altered fetal development of adiposity. Postnatal catch-up growth is a compensatory phenomenon, whose end is to restore normal balance. In this perspective, the degree of catch-up growth, which indeed affects the severity of insulin resistance in SGA subjects, can be viewed as a surrogate index of the fetal damage. Indeed, it has been shown in various clinical situations of starvation including reduced fetal growth, that the catch-up growth phenomenon is more efficient for the fat mass than for the fat-free mass $[29,30]$. The association between low birthweight and insulin resistance in adulthood might therefore be explained by the very peculiar dynamic changes in adiposity observed in this situation. The recently reported association between type 2 diabetes and the age at adiposity rebound points to the critical role of dynamic changes in the long-term metabolic outcome whatever the degree of adiposity $[31,32]$. It can be speculated that the altered fetal development of adiposity might permanently alter the regulation of its metabolic and hormonal functions predisposing to the later development of insulin resistance. Since thinness at birth encompasses many identified or unidentified causes, it remains to be clarified whether the mechanisms responsible for the long-term metabolic outcome are specific to some aetiologies or result from the dynamic process leading to thinness itself.

In summary, we have demonstrated in the present study that at 22 years of age MS is sevenfold more frequent in subjects born SGA than in subjects born AGA. Moreover, in this population, thinness at birth but not birthweight influences postnatal growth and the development of insulin resistance that plays a central role itself in MS associated with SGA. Taken together our results suggest that fetal dynamic changes in adiposity are involved in the long-term metabolic consequences of reduced fetal growth. Therefore, a better knowledge of fetal growth patterns leading to SGA seems critical in order to unravel the mechanisms responsible for the postnatal outcome.

Acknowledgements The authors acknowledge the contribution of C. Traband, M. Grolet, M. Cominotti (RN) and Dr J. L. Boerher and the laboratory staff at the Hospital of the city of Haguenau. This study was supported by grants from Pfizer (France) and the Institut National de la Santé et de la Recherche Médicale (National Institute for Health and Medical Research) (INSERM, "PROGRES" 2000 2002). 


\section{References}

1. Barker DJ, Hales CN, Fall CH, Osmond C, Phipps K, Clark PM (1993) Type 2 (non-insulin-dependent) diabetes mellitus, hypertension and hyperlipidaemia (syndrome $\mathrm{X}$ ): relation to reduced foetal growth. Diabetologia 36:62-67

2. Valdez R, Athens MA, Thompson GH, Bradshaw BS, Stern MP (1994) Birthweight and adult health outcomes in a biethnic population in the USA. Diabetologia 37:624-631

3. Eriksson JG, Forsen T, Tuomilehto J, Jaddoe VW, Osmond C, Barker DJ (2002) Effects of size at birth and childhood growth on the insulin resistance syndrome in elderly individuals. Diabetologia 45:342-348

4. Hales CN, Barker DJ, Clark PM et al (1991) Foetal and infant growth and impaired glucose tolerance at age 64. BMJ 303: 1019-1022

5. McCance DR, Pettitt DJ, Hanson RL, Jacobsson LT, Knowler WC, Bennett PH (1994) Birth weight and non-insulin dependent diabetes: thrifty genotype, thrifty phenotype, or surviving small baby genotype? BMJ 308:942-945

6. Lithell HO, McKeigue PM, Berglund Mohsen R, Little UB, Leon DA (1994) Relation of size at birth to non-insulin dependent diabetes and insulin concentrations in men aged 50-60 years. BMJ 312:406-410

7. Léger J, Lévy-Marchal C, Bloch J et al (1997) Reduced final height and indications for insulin resistance in 20 year olds born small for gestational age: regional cohort study. BMJ 315:341347

8. Jaquet D, Gaboriau A, Czernichow P, Lévy-Marchal C (2000) Insulin resistance early in adulthood in subjects born with intrauterine growth retardation. J Clin Endocrinol Metab 85:14011406

9. Flanagan DE, Moore VM, Godsland IF, Cockington RA, Robinson JS, Phillips DI (2000) Foetal growth and the physiological control of glucose tolerance in adults: a minimal model analysis. Am J Physiol Endocrinol Metab 278:E700-E706

10. Bavdekar A, Yajnik CS, Fall CH et al (1999) Insulin resistance syndrome in 8-year-old Indian children: small at birth, big at 8 years, or both? Diabetes 48:2422-2429

11. Reaven G (1988) Role of insulin resistance in human disease. Diabetes 37:1595-1607

12. DeFronzo RA, Ferrannini E (1991) Insulin resistance. A multifaceted syndrome responsible for NIDDM, obesity, hypertension, dyslipidemia, and atherosclerotic cardiovascular disease. Diabetes Care 14:173-194

13. Karlberg J, Albertsson-Wikland K (1985) Growth in full-term small for gestational age infants: from birth to final height. Pediatr Res 38:733-739

14. Ong KK, Dunger DB (2002) Perinatal growth failure: the road to obesity, insulin-resistance and cardio-vascular disease in adults. Best Pract Res Clin Endocrinol Metab 6:191-207

15. Papiernik E, Bouyer J, Dreyfus J et al (1985) Prevention of preterm births: a perinatal study in Haguenau, France. Pediatrics $76: 154-158$

16. Katz A, Nambi SS, Mather K et al (2000) Quantitative insulin sensitivity check index: a simple, accurate method for assessing insulin sensitivity in humans. J Clin Endocrinol Metab 85: $2402-2410$
17. Executive Summary of The Third Report of The National Cholesterol Education Program (NCEP) (2001) Expert panel on detection, evaluation, and treatment of high blood cholesterol in adults (adult treatment panel III). JAMA 285:2486-2497

18. Karpino BD (1958) Blood pressure and its relation to height, weight, race and age: World War II. Am J Hyg 68:288-311

19. Master AM, Lasser RP (1958) Relationship of the blood pressure to weight, height and body build in apparently healthy subjects, 65-196 years of age. Am J Med Sci 235:278-289

20. World Health Organization (1999) Definition, diagnosis and classification of diabetes mellitus: report of a WHO consultation. World Health Organization, Geneva, Switzerland, (Publication $\mathrm{WHO} / \mathrm{NCD} / \mathrm{NCS} / 99.2$ )

21. Li C, Johnson MS, Goran MI (2001) Effects of low birth weight on insulin resistance syndrome in Caucasian and African-American children. Diabetes Care 24:2035-2042

22. Okosun IS, Dever GE, Choi ST (2002) Low birth weight is associated with elevated serum lipoprotein(a) in white and black American children ages 5-11 y. Public Health 116:33-38

23. Phillips DI, Barker DJ, Hales CN, Hirst S, Osmond C (1994) Thinness at birth and insulin resistance in adult life. Diabetologia 37:150-154

24. Albertsson Wikland K, Wennergren G, Wennergren M, Vibergsson G, Rosberg S (1993) Longitudinal follow-up of growth in children born small for gestational age. Acta Paediatr 82:438-443

25. Veening MA, Van Weissenbruch MM, Delamarre-Van de Waal HA (2002) Glucose tolerance, insulin-sensitivity and insulin secretion in children born small for gestational age. J Clin Endocrinol Metab 87:4657-4661

26. Cianfarani S, Geremia C, Germani D, Scire G, Maiorana A, Boemi S (2001) Insulin-resistance and insulin-like growth factors in children with intrauterine growth retardation. Is catch-up growth a risk factor? Horm Res 55:7-10

27. Petersen S, Gotfredsen A, Ursin Knudsen F (1998) Lean body mass in small for gestational age and appropriate for gestational age infants. J Pediatr 113:886-889

28. Lapillonne A, Braillon P, Claris O, Chatelain PG, Delmas PD, Salle BL (1997) Body composition in appropriate and small for gestational age infants. Acta Paediatr 86:196-200

29. Hediger ML, Overpeck MD, Kuczmarski RJ, McGlynn A, Maurer KR, Davis WW (1998) Muscularity and fatness of infants and young children born small- or large for gestational age. Pediatrics 102:1-7

30. Dulloo AG, Jacquet J, Montani JP (2002) Pathways from weight fluctuations to metabolic diseases: focus on maladaptive thermogenesis during catch-up fat. Int J Obes Relat Metab Disord 26:S46-S57

31. Eriksson JG, Forsén T, Tuomilehto J, Osmond C, Barker DJP (2003) Early adiposity rebound in childhood and risk for type 2 diabetes in adult life. Diabetologia 46:190-194

32. Bhargava SK, Sachdev HS, Fall CH et al (2004) Relation of serial changes in childhood body-mass index to impaired glucose tolerance in young adulthood. N Engl J Med 350:865875 